\title{
HISTOLOGICAL PROFILE OF PATIENTS SERVED IN A REFERENCE CENTER IN THE CITY OF SÃO PAULO
}

Juliana da Costa Souza¹, Jamila Vieira de Sousa', Felipe Andreotta Cavagna', Jorge Yoshinori Shida', Luiz Henrique Gebrim

${ }^{1}$ Hospital Pérola Byington - São Paulo (SP), Brazil.

Introduction: Breast cancer is the most common cancer in women worldwide. In Brazil, according to INCA, it had an incidence of 57,900 new cases in 2018, 16,340 cases in the state of São Paulo. The WHO classifies tumors according to morphological criteria, including non-special breast cancer (CMI-SOE), which is seen in $70 \%$ of cases, with more than 20 other special histological types, among which invasive lobular carcinoma (ILC) has a higher prevalence (10-15\%). Objective: To describe the histological profile of breast cancer patients at the Pérola Byington Hospital between the years 2009 to 2019. Method: Cross-sectional, descriptive study, obtained through a database review. It included patients with suspected invasive neoplasms seen at the service. Results: During the period, 10,539 patients with malignant lesions and ductal carcinoma in situ (DCIS) were treated, among those who had cancer, 91\% were CMI-SOE, 5\% lobular neoplasia, and $3.95 \%$ of the other special subtypes. The least frequent type was metaplastic, with only 20 cases in the period. In addition, $7.14 \%$ of the patients seen had DCIS. Conclusions: It is possible to observe the predominance of CMI-SOE in this series, as well as described in analyses carried out throughout the country and in the world, followed by lobular carcinoma and other special subtypes. 